\title{
Reducing of Authentication Signaling Traffic in LTE Networks
}

\author{
Seonho $\mathrm{Kim}^{\dagger} \cdot$ Jongpil Jeong ${ }^{++}$
}

\begin{abstract}
As a result of the prevalence of smartphone, various mobile services became faster by LTE networks. Because many mobile devices are used more wireless services, heavy signaling traffic for authentication could be generated. Authentication is an important factor in wireless networks to identify devices; it is the start of wireless networks. This paper analyzes previous patterns for more effective authentication in accessing of another external networks. We propose a fast authentication scheme for minimizing of signaling cost between the authentication server and external networks. And we calculate the rate of authentication occurrence in LTE networks using mathematical modeling as well as the change of signaling cost for authentication in various network environments. Finally, we calculate the optimized number of authentication data and show the effectiveness for authentication signaling costs.
\end{abstract}

\section{Keywords : LTE(Long Term Evolution) Networks, EPS(Evolved Packet System), Authentication and Key Agreement, Mobile Network Security, Poisson Distribution}

\section{LTE 네트워크에서 인증 시그널링의 감소 기법}

\author{
김 선 호$^{+} \cdot$ 정 종 필 ${ }^{++}$
}

\begin{abstract}
요 약
현재 많은 스마트폰의 보급으로 LTE 네트워크를 통해 다양하고 빠른 모바일 서비스를 하게 되었다. 이에 수많은 모바일 기기들이 이전보다 더 많은 무선서비스를 이용하게 되고 그에 따른 수많은 인증 시그널링이 발생하게 된다. 이러한 무선네트워크에서 인증은 무선기기를 식별할 수 있는 중요한 역할을 하며 무선네트워크의 시작이기도 하다. 그래서 본 논문에서는 이전에 인증이 발생된 패턴을 분석하여 또 다른 외부 네 트워크로 접근할 때 이전 보다 효과적인 인증을 수행한다. 그래서 LTE 네트워크에서 외부 네트워크와 인증서버 사이의 시그널링 비용을 최소 화하여 빠른 무선 인증 서비스를 제안한다. LTE 네트워크에서 인증이 발생되는 비율을 수학적 모델링을 이용하여 계산하였고, 이를 다양한 환 경에서 인증 시그널링 비용의 변화를 계산하였다. 본 논문에서 제안한 최적 인증 데이터의 수를 계산하여 이전 보다 효율적인 인증 시그널링 비용이 발생함을 보인다.
\end{abstract}

키워드 : LTE(Long Term Evolution) 네트워크, EPS(Evolved Packet System), 인증과 키 동의, 모바일 네트워크 보안, 푸아송 분포

\section{1. 서 론}

요즘 많은 스마트 폰의 보급으로 모바일 유저들은 다 양한 서비스를 사용할 수 있게 되었다. 이런 모바일의 다 양한 서비스는 좀 더 빠른 무선 네트워크의 수요로 나타 났고, 통신사들은 LTE(Long Term Evolution) 서비스를 가입자에게 제공하게 되었다. $3 \mathrm{G}$ 에 비해 LTE의 주요 이 점은 높은 처리량, 낮은 지연 시간, 플러그앤 플레이, 같 은 플랫폼에서FDD(Frequency Division Duplex)와 TDD

※ 이 논문은 2011년도 정부(교육과학기술부)의 재원으로 한국연구재단의 기초 연구사업 지원을 받아 수행된 것임(2011-0027030)

† 준 회 원: 성균관대학교 정보통신대학 정보보호학과 공학석사

†† 정 회 원: 성균관대학교 산학협력단 산학협력중점교수

논문접수 : 2012년 6월 4일

심사완료 : 2012 년 8월 7일

* Corresponding Author: Jongpil Jeong(jpjeong@skku.edu)
(Time Division Duplex)를 사용할 수 있다는 점, 향상된 사용자 성능, 단순한 아키텍쳐, 그로 인한 낮은 운영비 등 이다. EPS(Evolved Packet System)는 LTE, HSDPA/ HSDPA+(High Speed Downlink Packet Acess)등 다양한 액세스 네트워크를 지원하는 All-IP 기반의 시스템이다. LTE는 EPS 기술을 이용하고 이는 $3 \mathrm{GPP}(3 \mathrm{rd}$ Gerneration Partnership Project)의 릴리즈 8을 기반하고 있으며 현재 릴리즈 10 까지 나왔다[8].

하지만 빠른 무선 네트워크를 제공하는 LTE에서의 EPS 기술은 불법의 접근, 악의적인 수정, 서비스 거부 공격 등의 이전보다 많은 위험에 노출되어 있다. 그래서 $3 \mathrm{GPP}$ 는 기존 의 $3 \mathrm{G}$ 망에서의 보안을 개선할 수 있는 정교한 키 계층 과 보안 컨텍스트 교환을 제공하는 새로운 보안 표준 발표했다 [1]. 강화된 $\mathrm{EPS}$ 네트워크에서 인증의 중요성은 매우 크다. 
무선 네트워크에서 인증은 외부 네트워크에 노출된 무선기 기의 익명성을 보장하면서 자신을 정보를 인증서버로부터 증명할 수 있게 한다. 이처럼 무선 네트워크에서 인증은 가 장 중요한 요소이면서 가장 많이 수행되는 서비스이다. LTE는 기존 HSDPA보다 12 배 이상 빠른 속도로 통신할 수 있다. 이에 더 많은 무선 네트워크 서비스를 사용하게 되었 고 그에 따른 이전 보다 더 많은 인증 시그널링이 발생 하 게 되었다. 하지만 $3 \mathrm{GPP}$ 의 $\mathrm{EPS}$ 기술에는 인증 시그널링 로 드를 평가하는 연구는 없었다. 그래서 본 논문에서는 가입 자의 인증 이벤트 패턴을 분석하고 그 가입자에 맞는 최소 의 인증 시그널링 비용을 발생시켜 외부 네트워크와 인증 서버간의 최소한의 인증 시그널링 비용을 발생시킬 수 있는 기법을 제안한다.

외부 방문 네트워크에 접근한 모바일 유저는 인증 이벤 트를 발생시키고 홈 네트워크에 있는 HSS(Home Subscriber Server)로부터 인증 데이터를 요청한다. 이때 홈 네트워크에 접근비용이 비싸기 때문에 인증 서버는 한 번에 여러 개의 인증 데이터를 방문 네트워크에 전송해 준다. 본 논문에서는 외부 네트워크에서 홈 네트워크에 있 는 HSS와의 접근을 최소화하여 트래픽비용을 줄이려한다. 그래서 HSS로부터 한 번에 여러 개의 인증데이터를 받아 적절한 수를 측정하여 방문 네트워크와 홈 네트워크의 접 근을 최소화한다.

LTE 네트워크에서의 인증은 방문 네트워크와 홈 네트워 크의 위치적인 문제점과 네트워크 방식의 차이점으로 외부 방문 네트워크와 인증서버(HSS)간의 인증 정보 요청 또는 전달에 있어 많은 네트워크 비용이 발생한다. 때문에 $3 \mathrm{GPP}$ 에서는 HSS에서 한 번에 여러 개의 인증 벡터를 보내어 외 부 네트워크에서 사용할 수 있게 한다. 3GPP의 TS 29.002 의 인증 정보 맵에서 $3 \mathrm{G}$ 의 인증 벡터 수는 5 로 고정되어 있다[2]. 이는 LTE 네트워크의 다양한 인증 이벤트 환경에 적합하지 않다. LTE 네트워크에서는 가입자가 인증 벡터 수를 HSS에게 요청할 수 있다. 그래서 본 논문은 HSS에게 인증 벡터 수를 요청할 때 최적의 인증 벡터 수를 요청하여 특정 외부 네트워크 내에서 사용하여 HSS와 외부 네트워크 간의 트래픽을 최소화하려고 한다. 가입자가 인증을 발생시 키는 비율을 다양한 수학적 모델링을 이용하여 다양한 환경 에서의 인증 발생 패턴을 분석한다. 분석한 인증 발생 패턴 을 기반으로 최적의 인증 벡터 수를 계산하여 최소의 인증 시그널링 비용을 발생시키도록 한다.

논문의 구성은 다음과 같다. 관련연구에서는 이전의 다 양한 모바일 네트워크 인증 기법과 LTE 네트워크 시스템, $\mathrm{LTE}$ 에서 사용하는 $\mathrm{EPS}$ 기술의 인증(EPS-AKA) 절차에 대해 기술했다. 그리고 제안 기법에서는 수학적 모델링을 통해 LTE에서의 인증 시그널링 비용을 수식으로 표현한 다. 그 수식을 통해 본 논문에서 제안하는 기법으로 최적 의 인증데이터 수를 계산하여 최소의 비용을 분석하고 증 명한다.

\section{2. 관련 연구}

\section{1 모바일 네트워크 인증}

$3 \mathrm{GPP}$ 에서의 인증서버는 모바일 네트워크 인증과 키 동의 가 발생할 때 한 번에 여러 개의 인증 벡터들을 발생시키고 방문한 외부 네트워크(visited network)에 여러 개의 인증 벡터들을 한 번에 전달한다. 이 기술은 방문한 외부 네트워 크와 인증서버 사이의 많은 시그널링 트래픽을 감소시킨다. 하지만 방문한 외부 네트워크에는 그 여러 개의 인증 벡터 들을 저장할 추가의 스토리지 비용이 발생하게 되었다[3][4]. 이전의 연구들에서 많은 분석 모델들은 이러한 전달되는 인 증 벡터 수의 중요성을 연구하였다[5][6]. Lin은 인증 벡터들 의 수를 이용하여 인증 시그널링 트래픽분석에 대한 최초의 연구를 시도했다[5]. 복잡하지 않은 알고리즘들을 통해 인증 벡터 수의 크기를 결정하여 인증 서버에 도움을 줄 수 있는 기법이 제안되었다. Al-Saraireh은 Lin의 기법은 최적의 시 그널링 트래픽 감소에 대한 알고리즘을 좀 더 개선했었다 [6]. Lin 과 Al-Saraireh의 분석적 모델링 방법론은 푸아송 도착 프로세스와 셀에 머무르는 시간 등에 대하여 지수 분 포를 기반으로 하는 기법에서 상당히 유사하다. 한편 Zhang 은 결국 이전에 사용되던 인증 벡터는 모두 사용해야지만 다음 인증 이벤트 때에 인증 서버로부터 새로운 인증 벡터 를 받을 수밖에 없다는 것에 주목했다[7]. 그들이 제안한 Pre-authentication 기법은 약간 증가된 시그널링 오버헤드 와 함께 인증 지연을 감소시키는 것을 보여준다. 최소한의 인증 시그널링 트래픽을 위해서는 적절한 인증 벡터 배열의 크기를 통해 최소 $\mathrm{HSS}$ 의 접근으로 전송 비용을 줄이는 것 이다. 이러한 이전의 연구들을 통해 최근의 LTE에서 사용 되는 $\mathrm{EPS}$ 기술의 인증(EPS-AKA)에 적합한 인증 시그널링 감소기법을 제안하려 한다.

\subsection{LTE Networks System}

LTE 네트워크에서는 $3 \mathrm{G}$ 기술에서 발전한 다양한 요소들 이 있다. Table 1에서 LTE 네트워크에서의 약어들을 명시 하였고, Fig. 1은 가입자가 이동하면서 새로운 외부 네트워 크에 접근하였을 때 유저 데이터의 라우팅에 대한 LTE 네 트워크 시스템 및 보안 기술을 나타내었다[1][8].

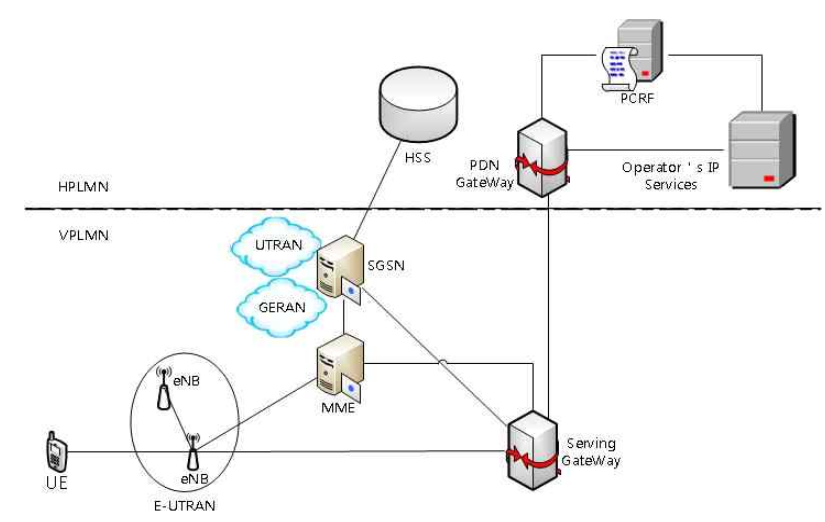

Fig. 1. LTE network system 
Table 1. Acronyms for the LTE system

\begin{tabular}{|c|l|}
\hline 약어 & \multicolumn{1}{|c|}{ 설명 } \\
\hline AKA & Authentication and Key Agreement \\
\hline ASME & Access Security Management Entity \\
\hline AuC & Authentication Center \\
\hline AUTN & Authentication Token \\
\hline AV & Authentication Vector \\
\hline eNB & Evolved Node B \\
\hline EPS & Evolved Packet System \\
\hline E-UTRAN & $\begin{array}{l}\text { Evolved Universal Terrestrial Radio } \\
\text { Access Network }\end{array}$ \\
\hline HPNMN & Home Public Land Movile Network \\
\hline HSS & Home Subscriber Server \\
\hline MME & Mobility Management Entity \\
\hline PLMN & Pubic Land Mobile Network \\
\hline RAND & RANDom number \\
\hline RES & Response \\
\hline UE & User Equipment \\
\hline UICC & Universal IC Card \\
\hline USIM & Universal Subscriber identity Module \\
\hline VPLMN & Visted Public Land Mobile Network \\
\hline
\end{tabular}

LTE 네트워크에서 가장 중요한 세 가지 개념은 HPNMN(Home Public Land Movile Network), VPLMN (Visted Public Land Mobile Network) 그리고 UE(User Equipment)이다. 모바일 사용자는 UE로 표기 되며, UE는 $\mathrm{ME}$ (Mobile Equipment)와 가입자 모듈 (UICC/USIM)으로 구성되어진다.

1) HSS(Home Subscriber Server) : HSS는 UMTS (Universal Mobile Telecommunication System)의 HLR (Home Location Register)에서 발전한 것으로 홈 네트워 크(HPLMN)에서 가입자 정보를 관리하는 중앙 집중화된 데이터베이스이다. HSS는 사용자의 등록/변경 관리, 인증 권한 부여, 로케이션, 세션 라우팅, 과금 등의 호/세션 제 어를 위한 모든 가입자 정보를 관리한다. LTE 네트워크 시스템에서 HSS는 EPS-AV(EPS Authentication Vector) 라고 불리는 인증서를 $\mathrm{EPS}-\mathrm{AKA}$ 프로토콜에 의해 $\mathrm{MME}$ 에게 전달한다.

2) MME(Mobility Management Entity) : UMTS에서의 $\mathrm{SGSN}$ 에서 발전한 $\mathrm{MME}$ 는 $\mathrm{SGSN}$ 과 마찬가지로 방문한 외부 네트워크(VPLMN)에 위치하며, EPS-AKA 프로토콜 에서의 시도-응답에 대한 네트워크 종단이다. 사용자 인증 과 사용자 프로파일의 다운로드를 위하여 $\mathrm{HSS}$ 와 통신하 고, NAS(Non Access Stratum) 시그널링을 통해 UE에게 $\operatorname{EMM}(\mathrm{EPS}$ Mobility Management) 및 ESM(EPS Session Management)기능을 제공한다. 또한 $\mathrm{UE}$ 는 처음 무선 네 트워크에 연결을 시도할 때 $\mathrm{MME}$ 로부터 인증을 거쳐야 한다. 인증을 통한 연결 후에도 $\mathrm{MME}$ 는 지속적으로 인증 을 요구할 수 있다. 그리고 $\mathrm{MME}$ 는 $\mathrm{UE}$ 의 위치를 기록하 고 추적할 수 있는 이동성관리의 역할을 수행한다. $\mathrm{MME}$ 와 $\mathrm{UE}$ 사이의 시스템 시그널링 보안은 NAS 보안으로 가 능하다. NAS 보안은 UE와 MME간의 NAS 시그널링 메
시지에 대한 무결성(필수)과 암호화(선택)를 수행한다.

3) $\mathrm{eNB}(\mathrm{eNodeB})$ : $\mathrm{eNB}$ 는 LTE에서 무선 접근 포인트이고 접근한 외부 네트워크 (VPLMN)에 속해있다. 그리고 $\mathrm{eNB}$ 는 $\mathrm{AS}$ (Access Stratum) 보안의 네트워크 종단이다. $\mathrm{eNB}$ 는 $\mathrm{E}-\mathrm{UTRAN}$ 안에서의 코어 네트워크 쪽으로의 통 신을 보호해야 한다. $\mathrm{AS}$ 보안은 $\mathrm{eNB}$ 와 $\mathrm{UE}$ 간에 $\mathrm{RRC}$ (Radio Resource Control) 시그널링의 데이터 무결성 (필수)과 데이터 암호화(선택)을 수행하고 사용자에서는 $\mathrm{IP}$ 패킷에 대한 암호화(선택)를 수행한다.

4) 가입 모듈 (UICC/USIM) : UICC/USIM은 E-UTRAN의 접근하는 가입자 식별모듈이다. 이전 $\mathrm{GSM}$ (Global System for Mobile communications)의 SIM 스마트카드 또한 호환성의 이유로 UTRAN/UMTS로 접근이 허용되 어야 하지만 GSM의 SIM은 GSM 보안과 싱글 64-bit 암 호화 키 $(\mathrm{Ke})$ 때문에 제한되어진다. UMTS에서의 사용되 는 2개의 $128-\mathrm{bit}$ 키 $(\mathrm{CK}, \mathrm{IK})$ 에서 $64-\mathrm{bit}$ 키 $(\mathrm{Ke})$ 로 변경하 는 것은 암호학에서는 불가능한 일이기 때문에 GSM의 $\mathrm{SIM}$ 은 E-UTRAN의 접근을 허락하지 않는다.

5) 모바일 장비(ME) : UMTS에서 $\mathrm{ME}$ 는 오직 무선 암호에 대해서만 책임지고 있다. 하지만 LTE에서의 $\mathrm{ME}$ 는 무선 암호뿐만 아니라 UMTS-AKA에서 발전된 EPS-AKA 프 로토콜의 키 체계를 유도해야 한다.

\subsection{LTE 인증 절차}

UTMS과 LTE에서 인증서(Authentication Credentials)는 인증 벡터(AV)라고 한다. LTE에서는 $\mathrm{EPS}-\mathrm{AV}$ 라 하며, $\mathrm{UMTS}$ 에서와는 다르게 세션 키가 $\mathrm{K}_{\mathrm{ASME}}$ 마스터키로 대체되 었다[1]. Fig. 2는 EPS-AKA프로토콜을 이용한 EPS-AV 전 달을 보여준다.

LTE에서의 인증은 네트워크에 대한 모바일의 인증뿐만 아니라 모바일에서의 네트워크 인증을 지원한다. 그래서 LTE에서 인증은 홈 네트워크의 HSS 와 UE의 USIM등 2 개의 주요한 인증 프로세스가 있다. 인증 절차는 다음과 같 은 두 가지 절차로 나타낼 수 있다

- 인증 벡터 분배(Distribution of authentication Vector) : $\mathrm{HSS}$ 가 $\mathrm{MME}$ 에게 $\mathrm{EPS}-\mathrm{AV}$ 를 분배하는 절차이다.

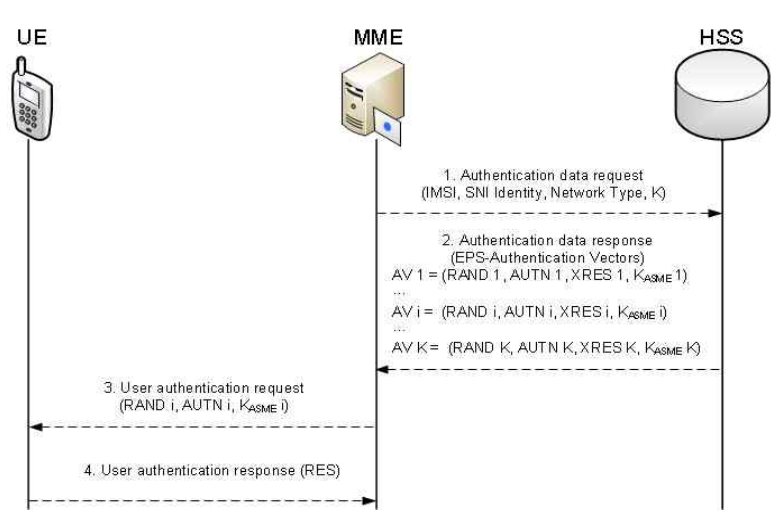

Fig. 2. LTE certification process 
$\mathrm{MME}$ 는 $\mathrm{HSS}$ 에게 $\mathrm{EPS}-\mathrm{AV}$ 의 수 $\mathrm{K}$ 를 요청하여 여러 개 의 $\mathrm{EPS}-\mathrm{AV}$ 를 $\mathrm{K}$ 의 수만큼 한 번에 받을 수 있다.

- 인증과 키 설정(Authentication and Key Establishment) $\mathrm{LTE}$ 에서 각각의 인증은 모바일과 $\mathrm{HSS}$ 가 보안키를 공유 하는 것에서부터 이루어진다. MME은 $\mathrm{HSS}$ 로부터 EPS$\mathrm{AV}$ 를 분배받고 해당 보안정보를 통해 모바일에게 인증 을 해줄 수 있는 것이다.

Fig. 2와 같이 LTE의 $\mathrm{EPS}-\mathrm{AKA}$ 인증 절차는 다음과 같 이 이루어진다[1].

1 단계) $\mathrm{UE}$ 가 새로운 $\mathrm{MME}$ 지역으로 이동할 때, $\mathrm{MME}$ 은 이전에 저장된 인증정보를 이용할 수 없다. 그래서 $\mathrm{MME}$ 는 $\mathrm{HSS}$ 에게 인증 데이터 요청메시지를 통해 인증 벡터 분배절 차를 수행한다. 이 메시지는 $\mathrm{UE}$ 의 고유한 식별 값인 IMSI(International Mobile Subscriber Identity), 가입자가 접속한 망을 나타내는 $\mathrm{SN} \mathrm{ID} \mathrm{값,} \mathrm{인증} \mathrm{벡터의} \mathrm{수} \mathrm{K}, \mathrm{UE}$ 가 접속한 망의 종류(Networks Type)을 포함한다.

2단계) $\mathrm{MME}$ 로부터 받은 IMSI는 $\mathrm{HSS}$ 에 기록된 $\mathrm{UE}$ 와 식별하기 위해 사용한다. 식별을 통해 $\mathrm{HSS}$ 가 발생된 데이 터와 인증데이터 요청정보로 $\mathrm{HSS}$ 는 $\mathrm{MME}$ 에게 전송할 최상 위 레벨의 마스터키 $\mathrm{K}_{\mathrm{ASME}}$ 를 생성한다. $\mathrm{K}_{\mathrm{ASME}}$ 는 $\mathrm{SN}$ ID 값 을 이용하여 생성되기 때문에 접속한 망이 바뀔 때 마다 새 로운 $\mathrm{K}_{\mathrm{ASME}}$ 값을 생성한다. $\mathrm{HSS}$ 는 $\mathrm{K}_{\mathrm{ASME}}$ 를 이용하여 인증 벡터 $\mathrm{AV}=\left(\mathrm{RAND}, \mathrm{AUTH}, \mathrm{XRES}, \mathrm{K}_{\mathrm{ASME}}\right)$ 를 요청한 $\mathrm{K}$ 수만 큼 생성하여 인증 데이터 응답메시지를 통해 $\mathrm{MME}$ 에게 보 낸다. $\mathrm{AV}$ 는 랜덤 숫자인 RAND, 예상되는 응답 값XRES, 마스터 키 $\mathrm{K}_{\mathrm{ASME}}$, 그리고 인증 토큰 $\mathrm{AUTN}$ 으로 구성되어 있다.

3단계) $\mathrm{MME}$ 가 $\mathrm{UE}$ 의 인증을 시작할 때, $\mathrm{HSS}$ 로부터 전달 받은 정의된 인증 벡터 배열의 사용되지 않은 다음의 인증 벡터를 선택한다. 그리고 인증 요청 메시지를 통해 UE에게 $\mathrm{i}$ 번째 인증 벡터 RAND i, AUTN i과 KASME i를 보낸다.

4 단계) $\mathrm{UE}$ 는 $\mathrm{HSS}$ 에서 사용한 것과 같은 $\mathrm{EPS}-\mathrm{AKA}$ 알 고리즘을 통해 $\mathrm{AUTN}_{\mathrm{UE}}$ 값을 생성하고 $\mathrm{AUTN}$ i값과 비교 하여 LTE망을 인증한다. 그 후에 AKA 알고리즘을 통해 발 생된 $\mathrm{RES}$ 를 인증 응답 메시지를 통해 $\mathrm{MME}$ 에게 전달한다. $\mathrm{MME}$ 는 받은 RES를 $\mathrm{HSS}$ 로부터 받은 XRES와 비교한다. 만약 그들이 같다면, $\mathrm{UE}$ 의 인증은 성공적으로 완료된다[1].

\section{3. 제안 기법}

\section{1 인증 시간 다이어그램}

$\mathrm{UE}$ 가 이동하며 일어나는 인증 이벤트들을 시간 다이어그 램으로 표현하고 $\mathrm{EPS}-\mathrm{AV}$ 배열의 크기 $K$ 값이 인증 시그널 에 미치는 영향을 알아본다. 그리고 수학적 모델링을 이용 하여 EPS-AV 배열의 크기 $K$ 의 변화에 따른 인증 시그널 링 비용을 산출하고 최적의 $K$ 값을 산출한다.

Fig. 3의 시간 다이어그램에서 $\mathrm{UE}$ 가 새로운 $\mathrm{MME}$ 지역 을 시간 $\tau_{1,1}$ 에 들어간다고 가정한다. $\mathrm{UE}$ 는 $\mathrm{MME}$ 에게 등록 메시지를 보낸다. $\mathrm{MME}$ 가 인증정보를 가지고 있지 않기 때 문에 $\mathrm{ADR}$ 을 통해 인증 벡터를 전달받는 절차가 수행되어 지고, 이때 $\mathrm{HSS}$ 로부터 받은 인증 벡터들의 수는 $K$ 라고 한 다. MME는 $\mathrm{HSS}$ 로부터 인증 벡터 배열을 얻고 상호간의 인증은 $\mathrm{MME}$ 와 $\mathrm{UE} / \mathrm{USIM}$ 사이에 첫 번째 인증 벡터를 $\mathrm{UAR}$ 메시지를 통하여 사용한다[5]

시간 $\tau_{1,1}$ 후에, 두 번째 인증 이벤트는 시간 $\tau_{1,2}$ 에서 수 행되어진다. UE/USIM의 두 번째 UAR 이벤트가 시작되어 지고 $\mathrm{MME}$ 는 상호인증에 대해 인증 벡터 배열의 두 번째 인증 벡터를 사용한다. 시간 $\tau_{1, K}$ 에 $K$ 번째 인증 이벤트가 수행되어 지면서 인증 벡터 배열의 $K$ 번째 인증 벡터가 $\mathrm{UAR}$ 메시지에 의해 사용한다. 시간 $\tau_{1, K}$ 후에는 인증 벡 터 배열에 더 이상의 인증 벡터가 없으므로 다음 인증 이 벤트는 시간 $\tau_{2,1}$ 에서 새로운 인증 배열을 요청한다. $\mathrm{MME}$ 는 $K$ 번째 이벤트를 수행한 후 가능한 인증 벡터가 없는 것 을 인식할 때, HSS로부터 다음 인증 벡터 배열을 얻기 위 해 두 번째 $\mathrm{ADR}$ 절차가 수행되고 새로 받은 인증 벡터 배열의 인증 벡터들을 통해 다음 UAR이 수행한다. 다음 발생한 인증 이벤트들은 $\mathrm{ADR}$ 들과 $\mathrm{UAR}$ 들은 이미 설명했 던 것처럼 반복 수행한다. 시간 $\tau_{n+1}$ 때에는 $\mathrm{UE}$ 는 $\mathrm{MME}$ 지 역을 떠난다. 시간 $\tau_{n+1}$ 이전에 마지막 인증 이벤트는 시간 $\tau$ ${ }_{n, i}(1 \leq i \leq k)$ 때에 수행되어 지며, 인증 벡터 배열에서 $\mathrm{i}$ 번째 인증 벡터를 활용한다. 그리고 시간 $\tau_{n+1}$ 에 $K-i$ 만 큼의 나머지 인증 벡터 개수는 $\mathrm{MME}$ 에서 사용 되지 않는 다. 결국 $\tau_{n+1} \sim \tau_{1,1}$ 동안에 $(N-1) K+i$ 만큼의 $\mathrm{UAR}$ 과 $N$ 번의 $\mathrm{ADR}$ 들이 수행한다.

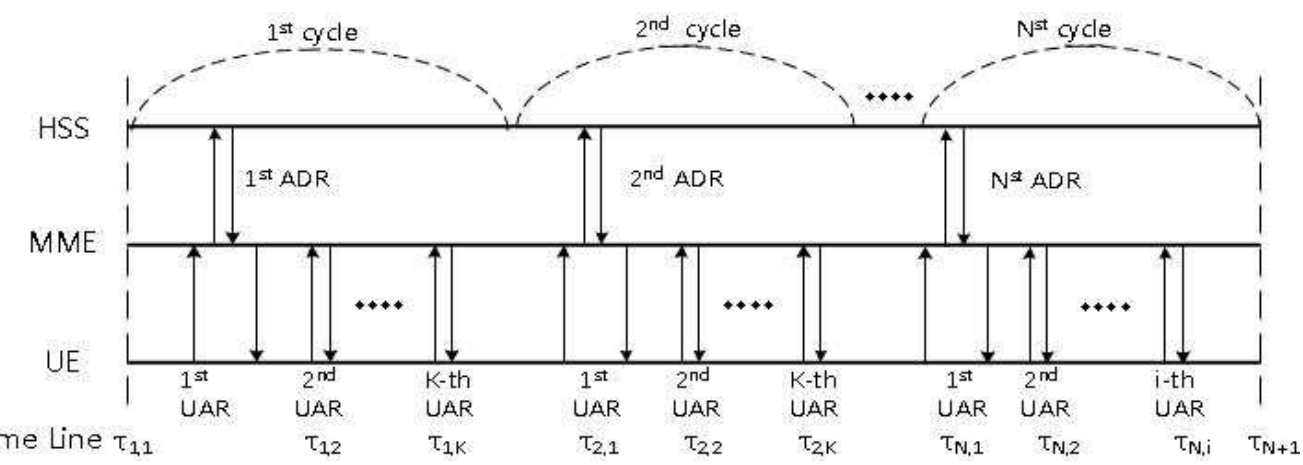

ADR : Authentication Data Request \& Response

UAR : User Authentication Request \& Response

Fig. 3. Timing Diagram 
$\mathrm{MME}$ 와 HSS는 다른 지역에 위치해 있는 경우가 많다. 그래서 $\mathrm{ADR}$ 명령은 비용이 많이 든다는 것을 알고 있다. 그러므로 $\mathrm{MME}$ 지역에서 $\mathrm{UE}$ 가 있을 때 $\mathrm{ADR}$ 들의 수행 숫 자를 감소시키기 위해 한 번에 많은 인증 벡터들을 가져와 야 한다. 즉 인증 벡터 배열 크기 $K$ 는 증가되어야 한다. 하 지만 큰 $K$ 값은 $\mathrm{MME}$ 에서 $\mathrm{HSS}$ 로부터 매번 인증 벡터들을 전송하는 동안 더 많은 네트워크 대역폭을 사용해야 한다. 이와 같이 인증 네트워크 시스널링 비용을 최소화하기 위한 적절한 $K$ 값을 선택해야만 한다. 다음 절에서는 분석적 모델 을 통해 인증 시그널링 비용을 최소화할 수 있는 최적의 $K$ 값을 계산할 수 있는 기법을 제안한다.

\section{$3.2 \mathrm{~K}$ 값에 따른 분석 모델링}

$n$ 은 $\mathrm{UE}$ 가 한 $\mathrm{MME}$ 지역에 있을 때의 $\mathrm{ADR}$ 이 수행되는 총 횟수이다. $\mathrm{HSS}$ 로부터 얻은 각각의 $\mathrm{ADR}$ 에 대한 인증 벡터들의 수를 $K$ 라고 한다. $\mathrm{UE}$ 가 특정 시간동안 $\mathrm{MME}$ 에서 일어나는 인증 이벤트를 푸아송 프로세스의 형식으로 제안 하였다. 특정한 기간 $\tau$ 동안 $\mathrm{UAR}$ 은 수행된다고 하면 $\Theta(n, K, \tau)$ 는 $\mathrm{HSS}$ 에서 $\mathrm{ADR}$ 이 $n$ 번 일어날 확률이다. 만약 특정기간 $\tau$ 에서 $(n-1) K+k(1 \leq k \leq K)$ 만큼 $\mathrm{UAR}$ 이벤트가 발생한다면 $n$ 번의 $\mathrm{ADR}$ 이 수행될 것이다. 푸아송 분포의 확률에 따르면 다음과 같이 표현된다.

$$
\Theta(n, K, \tau)=\sum_{k=1}^{K}\left\{\frac{(\lambda \tau)^{(n-1) K+k}}{[(n-1) K+k] !}\right\} e^{-\lambda t}
$$

$t$ 는 $\mathrm{UE}$ 가 한 $\mathrm{MME}$ 지역에 머무는 시간이다. Fig. 3에 의 하면 $t=\tau_{N+1}-\tau_{1,1}$ 로 표현될 수 있다. 시간 $t$ 가 밀도함 수 $f(t)$, 평균 $1 / \mu$, 그리고 라플라스 변환식 $f^{*}(s)=\int_{t=0}^{\infty} f(t) e^{-s t} d t$ 에 대한 분포를 가지고 있다 고 가정한다. 다음 수식 (2)의 $P(n, K)$ 는 $\mathrm{UE}$ 가 $\mathrm{MME}$ 지역 에 머무르는 동안의 $\mathrm{ADR}$ 이 $n$ 번 일어날 확률을 나타낸다.

라플라스 변환식 방법을 이용하여 수식 (2)에서 (3)을 도 출하였다. $E[N]$ 은 한 $\mathrm{MME}$ 서비스 지역에서 $\mathrm{UE}$ 가 머무르 는 동안 예상되는 $\mathrm{ADR}$ 의 이벤트 횟수이다. 그리고 다음과 같이 나타내었다.

$$
E[N]=\sum_{n=1}^{\infty} n P(n, K)
$$

다음과 같이 3 개의 $\mathrm{MME}$ 에 머무르는 시간 분포들을 기 반으로 $P(n, K)$ 과 $E[N]$ 을 도출하였다.

감마 분포(Gamma Distribution) : 만약 $f(t)$ 가 평균 $1 / \mu$ 이고 분산 $v$ 라면 다음과 같다.

$$
\begin{aligned}
& f^{*}(s)=(1+\mu v s)^{-\left(1 / \mu^{2} v\right)} \\
& \frac{d^{\iota} f^{*}(s)}{d s^{\iota}}=(-\mu v)^{\iota}\left[\prod_{j=0}^{\iota-1}\left(\frac{1}{\mu^{2} v}+j\right)\right](1+\mu v s)^{-\left(1 / \mu^{2} v+\iota\right)}
\end{aligned}
$$

특히 감마분포의 혼합은 거의 근사치에 가까운 양수의 확 률 변수를 보여준다. 또한 실제 모바일 네트워크에서 UE가 $\mathrm{MME}$ 에 머무르는 시간을 계산할 수 있다. 때문에 각각 다 른 $\mathrm{MME}$ 에 머무르는 시간 분포를 나타나기 위해 감마 분포 는 사용하기 충분하다[5]

수식 (4)와 (2)을 다시 나타내면 다음 수식(5)와 같다.

하이퍼-얼랑 분포(Hyper-Erlang Distribution) : 실수가 아닌 확률 변수의 확률 분포를 위한 매우 좋은 근사치에 가 까울 수 있는 또 다른 분포는 Hyper-Erlang 분포이다. 다음 과 같이 도출할 수 있다.

$$
E[t]=\frac{1}{\mu}=\sum_{i=1}^{I}\left(\frac{\beta_{i} m_{i}}{\mu_{i}}\right)
$$

여기서 $m_{i}$ 는 양의 정수이다.

$$
\beta_{i \geq 0} \text { 그리고 } \sum_{i=1}^{I} \beta_{i}=1
$$

Hyper-Erlang 분포의 라플라스 변환식으로 변환하면 다 음 수식(6)과 같다.

$$
\begin{aligned}
P(n, K) & =\int_{t=0}^{\infty} \Theta(n, K, t) f(t) d t \\
& =\sum_{k=1}^{K} \int_{t=0}^{\infty}\left\{\frac{(\lambda t)^{(n-1) K+k}}{[(n-1) K+k] !}\right\} e^{-\lambda t} f(t) d t \\
& =\sum_{k=1}^{K}\left\{\frac{\lambda^{(n-1) K+k}}{[(n-1) K+k] !}\right\} \int_{t=0}^{\infty} t^{(n-1) K+k} \times f(t) e^{-\lambda t} d t \\
& =\sum_{k=1}^{K}\left\{\frac{\lambda^{(n-1) K+k}}{[(n-1) K+k] !}\right\}(-1)^{(n-1) K+k} \times\left.\left[\frac{d^{(n-1) K+k} f^{*}(s)}{d s^{(n-1) K+k}}\right]\right|_{s=\lambda}
\end{aligned}
$$




$$
\begin{gathered}
P(n, K)=\sum_{k=1}^{K} \frac{(\lambda \mu v)^{(n-1) K+k}}{[(n-1) K+k] !} \times\left\{\left[\prod_{j=0}^{(n-1) K+k-1}\left(\frac{1}{\mu^{2} v}+j\right)\right] \times(1+\mu v \lambda)^{-\left[1 / \mu^{2} v+(n-1) K+k\right]}\right\} \\
f^{*}(s)=\sum_{i=1}^{I} \beta_{i}\left(1+\frac{s}{m_{i} \mu_{i}}\right)^{m_{i}} \\
P(n, K)=\sum_{i=1}^{I} \beta_{i}\left\{\left[\frac{\left(m_{i-1}\right) ! \mu_{i}^{m_{i}}\left(m_{i \lambda}\right)^{(n-1) K}}{\left(m_{i} \lambda+\mu_{i}\right)^{(n-1) K+m_{i}}}\right] \times\left[\sum_{k=1}^{K}\left(\frac{(n-1) K+k+m_{i}-1}{m_{i}-1}\right)\left(\frac{m_{i} \lambda}{m_{i} \lambda+\mu_{i}}\right)^{k}\right]\right\}
\end{gathered}
$$

여기서 수식 (6), (2)을 다시 쓰면 다음 수식(7)과 같다.

지수 분포(Exponential Distribution) : 수식 (5)에서 $\mu^{2} v=1$ 거나 (7)에서 $I=1, m_{i}=1$ 라고 할 경우, $t$ 는 지수 분포를 이용하여 수식 (2)를 다음과 같이 나타낼 수 있다.

$$
P(n, K)=\left(\frac{\lambda}{\lambda+\mu}\right)^{(n-1) K}\left[1-\left(\frac{\lambda}{\lambda+\mu}\right)\right]
$$

또한 (3)도 다음과 같이 나타낼 수 있다.

$$
E[N]=\frac{1}{1-\left(\frac{\lambda}{\lambda+\mu}\right)^{K}}
$$

지수 분포는 모바일 네트워크에서 MME가 머무르는 실 제 시간을 상세히 계산할 수 없다. 그러나 이 분포는 평균 값 분석에 대해 결과가 정확하고, 시스템의 성능 추정할 수 있다[5].

$C(K)$ 는 한 $\mathrm{MME}$ 지역에서 $\mathrm{UE}$ 가 머무를 때 $\mathrm{ADR}$ 들의 총 메시지 전송 비용을 나타낸다. 그리고 다음과 같이 나타 낼 수 있다.

$$
C(K)=E[N] \times(K+2 \alpha)
$$

여기서 $\alpha$ 는 인증 절차를 통해 발생되는 인증 벡터 전송 비용과 인증 벡터가 발생되는 시간에 대한 정규화된 하나의 시그널링 메시지 오버헤드에 대한 비용을 나타내었다. 이 오버헤드는 하나의 $\mathrm{ADR}$ 에서 요청과 응답 메시지를 한 쌍 으로 교환 하도록 고려되어 졌다. 수식 (8)와 (9)로부터 $\mathrm{MME}$ 에 머무르는 시간을 지수로 나타낸 것에 대한 총 $\mathrm{ADR}$ 비용을 다음과 같이 나타내었다.

$$
C(K)=\frac{K+2 \alpha}{1-\left(\frac{\lambda}{\lambda+\mu}\right)^{K}}
$$

다음의 성능평가에서 위에서 언급한 수학적 모델링을 그 래프로 나타내어 다양한 $K$ 값과 $\mathrm{UE}$ 가 $\mathrm{MME}$ 에 머무르는 시 간 분포 변화에 대한 인증 시그널링 비용을 비교해 본다. 또한 이전 $\mathrm{MME}$ 서비스 지역에서의 $\mathrm{UE}$ 의 행동 패턴을 분 석하여 인증 벡터 배열 크기인 최적의 $K$ 을 계산할 수 있도 록 한다. 이러한 최적의 $K$ 값은 $\mathrm{MME}$ 서비스 지역을 이동할 때 마다 최적의 $K$ 값을 계산하여 매번 최적화된 인증 시그 널링 비용을 나타낼 수 있게 된다.

\section{4. 성능평가}

\section{$4.1 \mathrm{~K}$ 값에 따른 인증 시그널링 비용}

이 단락에서는 이전의 수학적 모델링을 통해 인증 벡터 배열 크기 $K$ 값에 대한 예상되는 $E[N]$ 값과 비용 $C(K)$ 값 영향에 대해 보여준다. 인증 벡터 배열 크기 $K$ 값은

\begin{tabular}{|c|c|}
\hline Variables & Contents \\
\hline$K$ & Size of authentication vector array \\
\hline$k$ & $\begin{array}{l}\text { The rest number of authentication vectors } \\
\text { used before MME's service area leaving } \\
(1 \leq k \leq K)\end{array}$ \\
\hline$n$ & The number of ADR \\
\hline$M$ & The total number of UAR \\
\hline$\lambda(\lambda=1 / \mu)$ & $\begin{array}{l}\text { Various UAR (authentication events) } \\
\text { occurrence rate }\end{array}$ \\
\hline$C(K)$ & $\begin{array}{l}\text { Total message transmission cost for ADRs } \\
\text { when an UE resides in an MME area }\end{array}$ \\
\hline$E[N]$ & $\begin{array}{l}\text { The expected number of ADRs when the } \\
\text { MS resides in an MME service area }\end{array}$ \\
\hline$P(n, K)$ & $\begin{array}{l}\text { The probability that there are } \mathrm{n} \text { ADRs } \\
\text { during the UEs residence in the MME area }\end{array}$ \\
\hline$L(K)$ & $\begin{array}{l}\text { The discarded authentication vector } K \\
\text { number(loss) in an MME service area } \\
\text { leaving. }\end{array}$ \\
\hline
\end{tabular}
$\mathrm{MME}$ 가 HSS에게 요청 메시지를 보낼 때 그 크기에 대한 값을 보내고 $\mathrm{HSS}$ 는 $\mathrm{MME}$ 가 요청한 수만큼의 인증 벡터들 을 보내준다. 이전 단락에서의 분석 모델링은 특정 $K$ 값에 대한 $E[N]$ 과 $C(K)$ 을 계산할 수 있다. Table 2는 성능 평가에서 사용된 변수 값이다.

Table 2. Table for calculating the variable signaling costs 


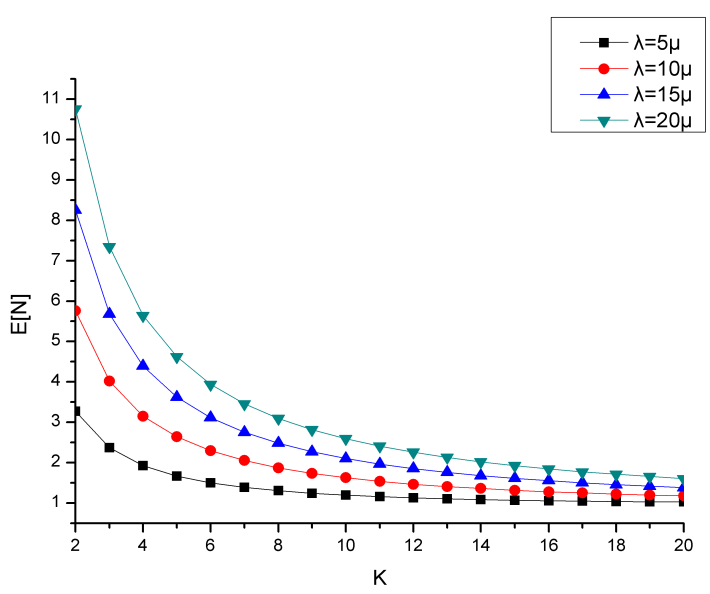

Fig. 4. Effect of UAR occurrence rate $\lambda$

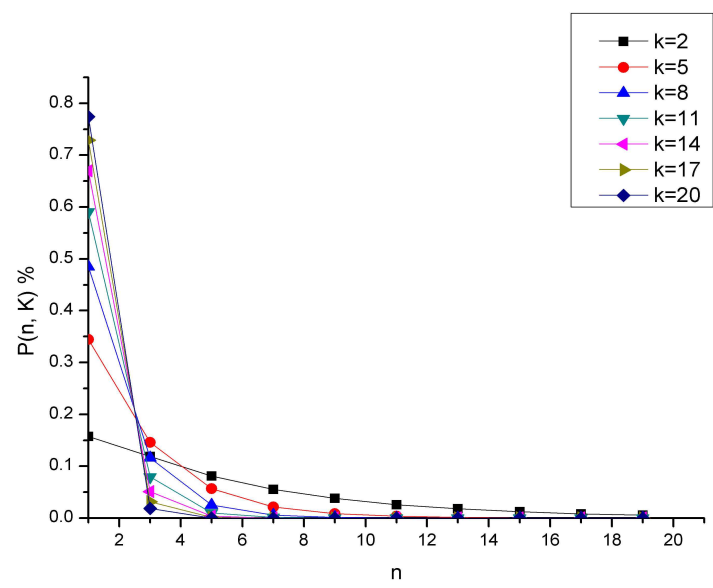

Fig. 5. The probability that ADR occurs $n$ times

Fig. 4는 다양한 $\mathrm{UAR}$ (인증 이벤트) 발생 비율 $\lambda$ 와 $K$ 값 에 대한 $E[N]$ 의 그래프이다. $\mathrm{MME}$ 에서 머무르는 시간들 은 평균 $1 / \mu$ 의 지수적인 분포로 추정한다. Fig. 4 에서 보듯이 $K$ 값이 10 보다 작을 때 UAR의 발생비율이 작을수록 $\mathrm{ADR}$ 이 일어나는 횟수가 크게 차이나는 것을 볼 수 있다. 또한 $K$ 값이 10 보다 클때 $5 \mu$ 와 $20 \mu$ 사이의 $\mathrm{ADR}$ 이 일어나 는 횟수 $E[N]$ 값은 많은 차이가 없다. 인증 시그널링 비용 을 최소화하기 위해서는 홈 네트워크에 접근해야하는 $\mathrm{ADR}$ (Authentication Data Request \& Response)은 최소화 해야 한다. Fig. 4의 그래프는 UAR 발생 비율과 $K$ 값에 변 화에 따른 시그널링 비용을 나타내고 있다.

Fig. 5 그래프는 $P(n, K)$ 의 확률 분포이며, 여기서 $\lambda=10 \mu$ 이다. $P(n, K)$ 는 특정 $K$ 값에 대한 $\mathrm{ADR}$ 이 $n$ 번 발생할 확률에 대한 그래프이다. 그래프에서 $P(n, K)$ 분포 는 $K$ 가 11 이상이고 $\mathrm{ADR}$ 의 횟수 $n$ 이 8 이상일 때 거의 유사 하며, $K$ 가 8 이하일 때 매우 다른 것을 확인할 수 있다. 특 히 $\mathrm{ADR}$ 이 3 이하로 발생할 확률에서 $K$ 값 2 와 20 의 차이는 매우 크다. 이것은 Fig. 4와 유사한 결과를 가지고 있다. $P(n, K)$ 과 마찬가지로 $E[N]$ 값은 큰 $K$ 값에 대한 결과가
유사하고 이는 $K$ 값의 증가는 시그널링 비용을 개선시키지 못한다는 의미이다.

Fig. 3에서 $(n-1) K+k(1 \leq k \leq K)$ 만큼 UAR 이 벤트가 일어난다고 가정한다. 이중에서 $K-k$ 값은 쓸 수없 는 인증 벡터로 버려지게 된다. 이는 새로운 $\mathrm{MME}$ 지역으 로 이동할 때 이전의 $\mathrm{MME}$ 에서 쓰다가 남은 인증 벡터를 쓸 수 없기 때문이다. 만약 매번 $\mathrm{ADR}$ 이벤트가 발생함에 따라 일정의 손실이 발생한다면, 그 손실은 계속해서 누적 되어 전체 네트워크 트래픽에서는 많은 영향을 미치게 된 다. 때문에 전체적인 네트워크 트래픽을 보았을 때에는 버 려지는 인증 벡터의 손실은 크게 보인다. 그렇기 때문에 $\mathrm{UAR}$ 발생에 따른 네트워크 트래픽 손실 여부를 간과할 수 없다. 나머지 버려지는 인증 벡터의 손실 값은 다음과 같이 나타낼 수 있다. 여기서 $\mathrm{k}$ 는 $1 \leq k \leq K$ 이고 $L$ 은 한 $\mathrm{MME}$ 지역에서 $n$ 번의 $\mathrm{ADR}$ 이 발생 했을 때의 손실 값이다.

$$
n K-(n-1) K+k=K-k=L(K)
$$

그리고 다음과 같이 $\mathrm{UAR}$ 이벤트 총 횟수를 $\mathrm{M}$ 이라 할 때 다음과 같이 나타낼 수 있다.

$$
\begin{aligned}
& M=(n-1) K+k \\
& M \equiv L \bmod K
\end{aligned}
$$

여기서 총 $\mathrm{UAR}$ 이벤트 횟수 $M=5, M=10, M=15, M=20$ 이라 하고 $K$ 값 변동에 따른 손실 값 $L$ 을 나타내었다. Fig. 6 에서는 UAR 횟수 대비 $K$ 값 증가에 따른 네트워크 손실 값 $L(K)$ 을 그래프로 그렸다. 그래프와 같이 손실 값은 일 정하게 증가하기 보다는 일정의 변동 폭이 있다. 다음과 같 이 UAR 횟수에 따른 서로 다른 최소의 손실을 나타내고 있고 $K$ 값의 증가에 따라 더 많은 손실 변동이 발생 되었다. 이런 인증 벡터 손실을 네트워크 비용으로 생각한다면 Fig. 6 역시 $K$ 의 증가는 오히려 네트워크의 비용을 더 증가시키 고 있다. 따라서 $\mathrm{UAR}$ 이벤트 수에 따라 최적의 $K$ 값과 인 증 시그널링 비용에 영향을 주게 된다.

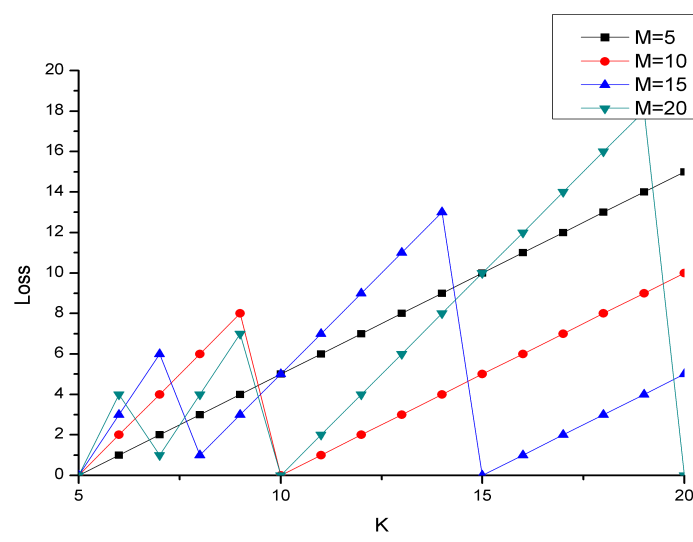

Fig. 6. For all UAR, Loss depends on changes in $K$ values 


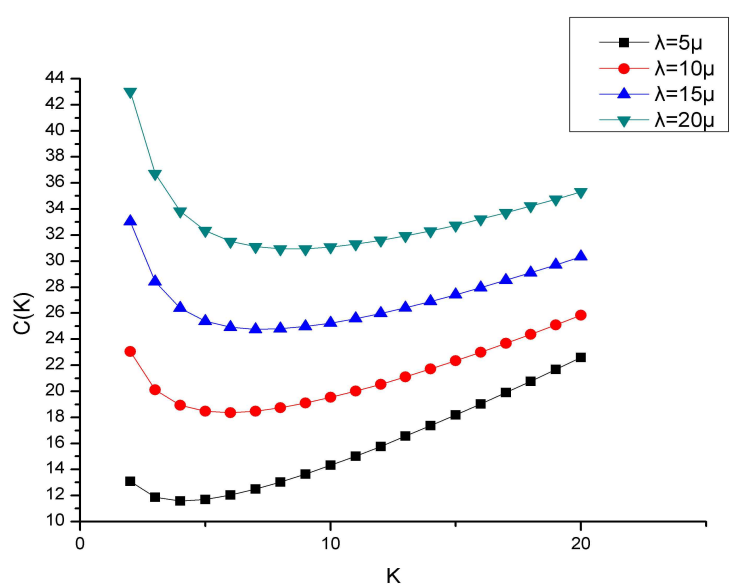

Fig. 7. The graph for $C(K)$ as a function of UAR rates

Fig. 7은 수식 (10)에서 $\alpha=1$ 일 때, 다양한 UAR 발생 비율 $\lambda$ 의 $\mathrm{K}$ 에 대한 $C(K)$ 값에 대한 그래프이다. 이는 $\mathrm{MME}$ 에서 머무르는 시간은 지수분포를 이용하였고 $K$ 값이 증가할 때 $C(K)$ 의 값에 대한 변동을 보여 주고 있다. 최 소 비용 $C(K)$ 에 대해 적절한 $K$ 값을 도출하기 위함이다. 또한 Fig. 7는 K값의 증가와 UAR이 발생될 비율 $\lambda$ 의 증가 는 시그널링 비용 $C(K)$ 의 증가를 가져왔다. 그 결과 $\mathrm{UAR}$ 이 발생될 비율 $\lambda$ 의 증가에 따른 그래프들은 $K$ 값에 대한 변동에 대해 $C(K)$ 가 영향을 받음을 알 수 있다. 따라서 $\mathrm{MME}$ 가 머무르는 시간 분포는 최소의 비용을 위한 인증 벡 터 배열 크기 값 $K$ 에 많은 영향을 준다.

4.2 최적의 인증 벡터 배열 크기

$3 \mathrm{GPP} \mathrm{TS} 29.002$ 에서의 $3 \mathrm{G}$ 의 인증 벡터 배열값이 $K=5$ 로 모바일 기술 맵에 설정되어 있다[2]. 하지만 인증 벡터 배열 값이 고정적으로 되어있는 것은 효율적이지 못하 다. 이전 성능 평가에서 UAR 트래픽에 따라 가장 최적의 $K$ 값이 변화가 있는 것이 확인하였다. 따라서 $\mathrm{UE}$ 의 $\mathrm{UAR}$ 트래픽 패턴 기반으로 $K$ 값을 동적인 선택을 할 수 있는 기 법을 제안한다. 이 기법은 $\mathrm{HSS}$ 또는 UE에서 실행되어 질 수 있다. 만약 이 기법이 UE에서 수행된다면, UE가 매번 새로운 $\mathrm{MME}$ 지역에 이동할 때 마다 새로운 $K$ 값 등록절차 에 의해 최적의 $K$ 값을 찾도록 수행되어 질 것이다. 그리고 $\mathrm{UE}$ 가 이전 $\mathrm{MME}$ 지역으로 부터 이동하여 새로운 $\mathrm{MME}$ 서 비스 지역 이동할 때 이전의 UE의 행동 패턴을 분석하여 최적의 $K$ 값을 찾고, 이를 $\mathrm{HSS}$ 에게 요청 하며 요청한 $K$ 값 만큼의 인증 벡터 배열을 전달해 주게 된다[1]. 최적의 인증 벡터 배열 크기 $K$ 는 $j$ 번째 새로운 $\mathrm{MME}$ 지역에서 $\mathrm{UE}$ 가 머 무르면서 $K$ 값을 측정하여 $j+1$ 번째에 요청한다고 하자. 이때 최적의 $K$ 값 측정은 다음과 같이 나타낼 수 있다.

측정 단계 : $j$ 번째 $\mathrm{MME}$ 서비스 지역에 머무르는 동안 $\mathrm{UAR}$ 의 이벤트 횟수 $M$ 을 측정한다.
결정 단계 : $\mathrm{UE}$ 가 $j$ 번째 $\mathrm{MME}$ 에 떠날 때, $K$ 값의 크기를 결정한다. 우리는 수식 (9)처럼 다음과 같은 계산방법을 따 른다. $L_{i}$ 는 수식 (11)을 따르며 이전에서 언급했던 인증 벡 터의 인증 벡터 손실을 시그널링 비용에 포함 시킨다. 인증 벡터 손실은 $K$ 값에 따라 $\mathrm{ADR}$ 이 발생 한 만큼 증가하여 전 체 네트워크 트래픽에 영향을 미칠 수 있기 때문에 인증 시 그널링 비용에 포함되어야 한다. 결정단계 시 그 수식은 다 음과 같이 표현된다.

$$
\begin{aligned}
& K_{1}=K(j)-2, \\
& K_{2}=K(j)-1, \\
& K_{3}=K(j) \\
& K_{4}=K(j)+1 \\
& K_{5}=K(j)+2 \\
& c_{i}=\left[\frac{M}{K_{i}}\right] \times K_{i}+L_{i}\left(n K_{i}-M\right), \text { for } i=1,2,3,4,5
\end{aligned}
$$

$K(j+1)=K_{m}$ where $1 \leq m \leq 5$ and $c_{m}=\min _{1 \leq i \leq 5}$

수식 (12)를 통해 수식 (13)처럼 최소의 $c_{i}$ 값을 가지는 최 적의 $K_{m}$ 값을 도출 해 낸다. $j+1$ 번째 $\mathrm{MME}$ 지역을 $\mathrm{UE}$ 가 방문했을 때 수식 (13)에서 측정 된 최적의 $K_{m}$ 값을 통 해 $\mathrm{HSS}$ 에게 인증 벡터 배열 크기를 요청한다. 그리고 $\mathrm{HSS}$ 는 요청 받은 $K$ 만큼의 인증 벡터를 전달한다.

Fig. 8에서 $\mathrm{MME}$ 에 머무르는 지수 분포는 $\lambda=10 \mu$ 이 고, $K(j)$ 는 이전의 인증 벡터 배열의 크기이다. 여기서 old_MME는 이전에 방문 했던 MME 서비스 지역이고 new_MME는 old_MME 이후에 방문된 MME 서비스 지역 이다. 그리고 $K(j+1)$ 은 수식 (12)과 (13)을 통해 얻어진 최적의 $\mathrm{K}$ 값으로 계산된 인증 시그널링 비용이다. 인증 시그

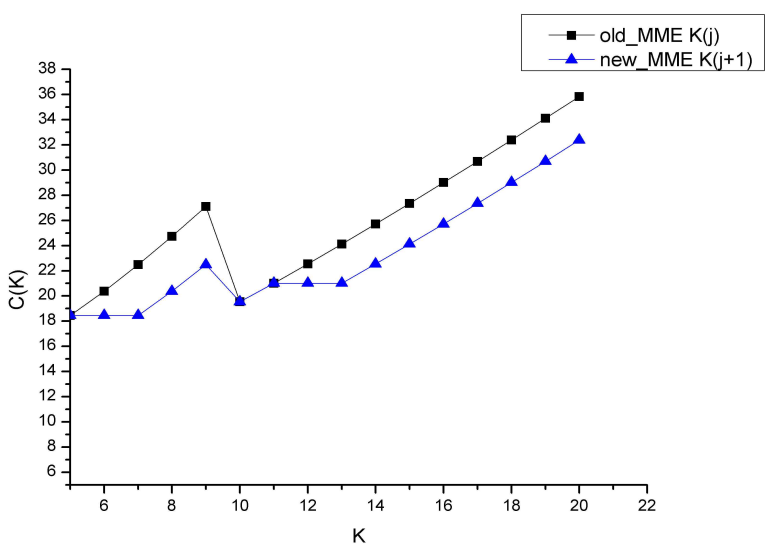

Fig. 8. Effect of reducing signaling cost as a function of $K$ 


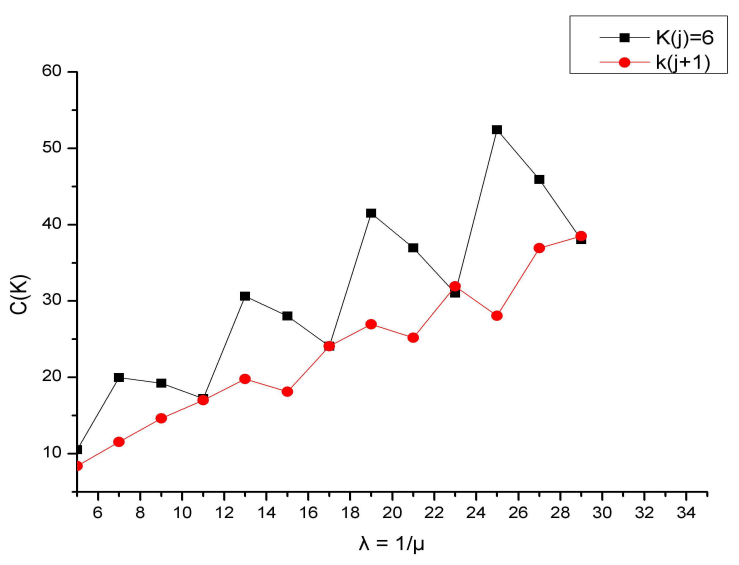

Fig. 9. Cost for optimal $K$ as a function of UAR occurrence rate $\lambda$

널링 비용은 Fig. 8과 Fig. 9에서도 마찬 가지로 이전의 수 식 (10)에서 (11)의 인증 벡터 손실 값을 더하여 시그널비용 을 계산하였다. Fig. 8에서 보듯이 새로운 MME 서비스 지 역을 방문하여 사용된 최적의 $K(j+1)$ 가 시그널 비용을 감소시킬 수 있는 것을 확인할 수 있다. 그래프 중간의 $K$ 값 이 5,10 의 경우 이전과 같은 시그널 비용을 나타내고 있다. 이는 이전의 $K$ 값이 이미 최적의 비용을 나타내므로 $K$ 값을 변경할 필요가 없음을 나타낸다.

Fig. 9은 인증 벡터 배열 크기를 6으로 고정한 환경에서 지수 분포 변화에 따른 시그널링 비용을 나타내었다. 그리 고 각각의 $K$ 값에 따라 최적의 $K$ 값을 계산하여 그에 따른 비용을 비교하여 나타내었다. Fig. 8과 마찬가지로 최적의 $K$ 값 계산으로 최소의 비용을 나타내고 있다. 또한 Table 3 에서 보듯이 $K$ 값이 $\lambda$ 의 증가에 따라 계속 증가 또는 감소 되지 않는 것을 확인할 수 있다.

이와 같은 성능평과들은 최적화한 $K$ 값의 선택이 최소의 인증 시그널링 비용을 가질 수 있음을 나타내고 있다. 그리 고 최소의 $K$ 값을 찾기 위해서는 이전의 $\mathrm{UE}$ 의 $\mathrm{UAR}$ 발생

Table 3. Change of $K$ for a given UAR occurrence rate $\lambda$

\begin{tabular}{|c|c|c|c|c|}
\hline$\lambda=1 / \mu$ & $K(j)$ & old_Cost & $K(j+1)$ & new_Cost \\
\hline 5 & 6 & 10.5247 & 5 & 8.359491 \\
\hline 7 & 6 & 19.95629 & 7 & 11.52635 \\
\hline 9 & 6 & 19.20782 & 5 & 14.65165 \\
\hline 11 & 6 & 17.21138 & 4 & 17.01067 \\
\hline 13 & 6 & 30.64492 & 7 & 19.7658 \\
\hline 15 & 6 & 28.03163 & 5 & 18.12885 \\
\hline 17 & 6 & 24.11076 & 6 & 24.11076 \\
\hline 19 & 6 & 41.52382 & 4 & 26.95508 \\
\hline 21 & 6 & 36.95324 & 7 & 25.18574 \\
\hline 23 & 6 & 31.06132 & 4 & 31.94145 \\
\hline 25 & 6 & 52.45948 & 5 & 28.07837 \\
\hline 27 & 6 & 45.909 & 4 & 36.93182 \\
\hline 29 & 6 & 38.03191 & 5 & 38.48128 \\
\hline
\end{tabular}

비율과 인증 벡터의 손실에 많은 영향을 받는 것을 확인하 였다. 따라서 본 논문에서 제안한 최적의 $K$ 값 측정 및 결정 으로 최소의 인증 시그널링 비용을 나타낼 수 있다.

\section{5. 결 론}

모바일 네트워크에서 인증은 $\mathrm{MME}$ 서비스 지역을 이동 하거나 매 인증 이벤트들 발생할 때 수행하게 된다. LTE 네트워크에서 각각의 인증은 $\mathrm{MME}$ 와 $\mathrm{UE}$ 에서 수행되며, 그 인증은 $\mathrm{HSS}$ 로부터 $\mathrm{MME}$ 가 전달받아 인증 데이터를 얻는 다. 이때, MME가 $\mathrm{HSS}$ 에 접근하는 비용이 비싸기 때문에 접근 수를 줄이기 위해 $\mathrm{MME}$ 는 한 번에 많은 크기의 인증 벡터를 얻어야 한다. 하지만 인증 벡터 배열에 대한 $K$ 의 크 기가 커지면, 즉 한 번에 많은 인증 벡터를 전달한다면 매 번 $\mathrm{HSS}$ 에서 $\mathrm{MME}$ 의 인증 벡터 배열 전송 비용이 더 비싸 지게 된다. 또한 하나의 $\mathrm{MME}$ 서비스 지역이 종료되었을 때 인증 벡터 배열의 크기가 커질수록 버려지는 인증 벡터 들의 수도 커져서 시그널링 비용의 낭비를 초래할 수 있다. 때문에 최소한의 인증 시그널링 비용을 위해 적절한 $K$ 값을 선택해야 한다. 여기서 제안한 분석 모델을 통하여 다음의 결과를 확인하였다.

$K$ 값의 증가는 $\mathrm{HSS}$ 에서 $\mathrm{MME}$ 로부터 접근하는 횟수 $E[N]$ 을 감소시킨다. 그러나 $K$ 가 커질 때 증가된 $K$ 는 오직 $E[N]$ 의 감소만을 나타내었고 인증 시그널링 비용 을 감소시키지는 못하였다.

- $\mathrm{UE}$ 가 $\mathrm{MME}$ 서비스 지역에 머무르는 시간이 증가할 때 $E[N]$ 은 증가한다. 그만큼의 인증 횟수가 증가하면서 $\mathrm{HSS}$ 로부터 인증 요청 횟수 $E[N]$ 도 증가하게 된다.

- 한 $\mathrm{MME}$ 서비스 지역에서 버려지는 인증 벡터의 시그널 링 손실은 인증 시그널링의 증가를 가져 온다.

- UE의 인증 이벤트 패턴에 맞는 적절한 $K$ 값은 인증 시 그널링 비용의 감소를 나타낸다.

인증 벡터 배열 크기 $K$ 을 조정하여 인증 트래픽의 값 $C(K)$ 을 줄여야 한다. 제안한 $\mathrm{MME}$ 가 머무르는 시간 분포 와 $\mathrm{UE}$ 의 $\mathrm{UAR}$ 트래픽 패턴, 인증 벡터의 손실을 기반으로 한 동적인 $K$ 값을 선택하는 기법을 제안한다. 적절한 $K$ 값은 네트워크 시그널링 비용을 감소시키는데 상당히 효과적이라 는 것을 보여준다. 누적된 네트워크의 전체 시그널링에 비 용에서 본다면 그 효과는 더욱더 효과적일 것이다. 본 논문 에서는 적절한 $K$ 값에 대해 이전의 $K$ 값과 개선된 $K$ 값의 성 능 결과를 비교하여 보여준다.

\section{참 고 문 헌}

[1] Third Gerneration Partnership Project; Technical Specification Group Services and System Aspects. 3GPP System 
Achitecture Evolution(SAE), "Security Architecure Security (Release 10)," 3GPP TS 33.401 version 10.2.0 September, 2011.

[2] Third Gerneration Partnership Project; Technical Specification Group Services and System Aspects. "Mobile Application Part (MAP) specification (Release 10)," 3GPP TS 29.002 version 10.6.0 March, 2012.

[3] Chung-Ming Huang and Jian-Wei Li, "Authentication and key agreement protocol for UMTS with low bandwidth consumption," Proceedings of the 19th International Conference on Advanced Information Networking and Applications (AINA), March, 2005.

[4] Ja' afer Al-Saraireh and Sufian Yousef, "A new authentication protocol for UMTS mobile networks," EURASIP Journal on Wireless Communications and Networking, Vol.2006, Issue 2, April, 2006.

[5] Yi-Bing Lin and Yuan-Kai Chen, "Reducing authentication signaling traffic in third-Generation mobile network," IEEE Transactions on Wireless Communications, Vol.2, No.3, May, 2003.

[6] Ja'afer Al-Saraireh and Sufian Yousef, "Analytical model for authentication transmission overhead between entities in mobile networks," Elsevier Computer Communications, Vol.30, Issue 8, June, 2007.

[7] Yan Zhang and Masayuki Fujise, "An improvement for authentication protocol in third-generation wireless networks," IEEE Transactions on Wireless Communications, Vol.5, No.9, September, 2006.

[8] Third Gerneration Partnership Project; Technical Specification Group Services and System Aspects. "General Packet Radio Service (GPRS) enhancements for Evolved Universal Terrestrial Radio Access Network(E-UTRAN) access, (Release 10),“ 3GPP TS 23.401 version 10.7.0 March, 2012.
[9] Yi-Bing Lin, Wei-Ru Lai, and Rong-Jaye Chen, "Performance analysis for dual band PCS networks," IEEE Trans. Comput., Vol.49, pp.148-159, Feb., 2000.

[10] Koien, G.M., "Mutual Entity Authentication for LTE," IEEE Trans Comput, pp.689-694, July, 2011.

[11] E. J. Watson, Laplace Transforms and Applications. Cambridge, MA

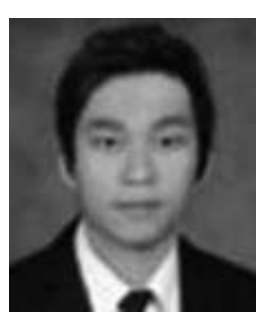

\section{김 선 호}

e-mail : pero5@skku.edu

2009년 안양대학교 디지털미디어공학 (공학사)

2011년 현 재 성균관대학교 정보통신 대학원 정보보호학과 공학석사 관심분야: 암호학, 인증과 키 동의, 모바일 네트워크

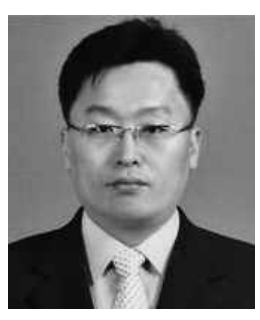

\section{정 종 필}

e-mail : jpjeong@skku.edu

2008년 성균관대학교 정보통신대학 (공학박사)

2009년 성균관대학교 컨버전스연구소 연구교수

2010년 현 재 성균관대학교 산학협력단 산학협력중점교수

관심분야: 모바일 컴퓨팅, 센서 이동성, 차량 모바일 네트워크, 스마트기기 보안, 네트워크 보안, IT 융합, 인터랙션 사이언스 등 